\title{
Lateral Concepts
}

\author{
CHRSTOPHER GAD \\ IT-UNIVERSITY OF COPENHAGEN \\ CASPER BRUUN JENSEN ${ }^{2}$ \\ OSAKA UNIVERSITY
}

\begin{abstract}
This essay discusses the complex relation between the knowledges and practices of the researcher and his/her informants in terms of lateral concepts. The starting point is that it is not the prerogative of the (STS) scholar to conceptualize the world; all our "informants" do it too. This creates the possibility of enriching our own conceptual repertoires by letting them be inflected by the concepts of those we study. In a broad sense, the lateral means that there is a many-to-many relation between domains of knowledge and practice. However, each specific case of the lateral is necessarily immanent to a particular empirical setting and form of inquiry. In this sense lateral concepts are radically empirical since it locates concepts within the field. To clarify the meaning and stakes of lateral concepts, we first make a contrast between lateral anthropology and Latour's notion of infra-reflexivity. We end with a brief illustration and discussion of how lateral conceptualization can re-orient STS modes of inquiry, and why this matters.
\end{abstract}

\section{Keywords}

infra-reflexivity; inter-reflexivity; lateral anthropology; lateral concepts

\section{Introduction}

Ever since ethnographic methods were first deployed in science and technology studies more than forty years ago, the field has been engaged in discussions about the relation between the knower and the known. Given that similar discussions have unfolded within anthropology and

. Christopher Gad, Email: chga@itu.dk

2Caper Bruun Jensen, Email: cbruunjensen@gmail.com

Copyright () 2016 (Christopher Gad and Casper Bruun Jensen). Licensed under the Creative Commons Attribution Noncommercial No Derivatives (by-nc-nd). Available at estsjournal.org. 
that STS is, in part, populated by anthropologists, the contact zone between these fields has gradually enlarged. Presently, ethnography operates simultaneously as a shared set of resources and as a domain of contestation in which knowledge production is being problematized in - and across - both fields. ${ }^{3}$

A key issue raised by ethnography is the complex relation between the knowledges (theories, concepts, assumptions) and practices of the researcher and those of the "informant." In anthropology, it is generally accepted that theories should not simply be "applied" as explanations of ethnographic settings but ought also in some sense to be shaped by those settings. But what is entailed by this requirement? How it can be operationalized? And with a view to what? These questions are far from settled.

An ethico-political impulse often motivates calls to let other modes of knowing and doing inform anthropological understandings. However, one can reach similar conclusions by a somewhat more descriptive route, by noticing that it is empirically the case that diverse forms of knowledge constantly spill over, modifying and inflecting one another.

This traffic of ideas and practices raises conceptual and methodological questions. The gambit of this essay is that, rather than trying to demarcate what properly belongs to STS (as theory) and what belongs to the empirical (and is thus data), we might enrich our conceptual repertoires and vocabularies by experimenting with these relations. The outcome is lateral concepts.

The premise of lateral concepts is that informants are "fellow travellers along the routes of social abstraction and analysis" (Maurer 2005: xv). Accordingly, there is no dichotomous or hierarchical relation between our concepts and informants' (Jensen 2014). The situation can be described as a Latourian "flat plane" on which all actors, researchers included, act and think. In this process, practices, theories and concepts move around laterally; they bump into each other, rub up against one another, and modify one another.

Latour's symmetrical plane is often said to ignore important differences, hierarchies, stratifications or forms of power. Let us therefore reiterate that it has nothing to do with the weird idea that everything is "equal." To the contrary, it has everything to do with creating a methodological position from which differences can be articulated and multiplied. A "flat"

"This is visible, for example, in ongoing, heated debates concerning ontology, taking place simultaneously in STS and in anthropology (Gad, Jensen and Winthereik 2015). 
starting point is required simply because we cannot in advance know what the relevant differences and subsequent asymmetries will turn out to be."

If "informants" are indeed "fellow travellers," it follows that at least some of their concepts and ideas hold potential for troubling, complicating or improving our own. We might thus say that the aim of lateral concepts is to simultaneously take into account insights developed in STS and the interpretations and forms of knowledge of informants.

This is not necessarily an easy task. For one, we often find ourselves confronted with notions that seem incompatible, or even incommensurable, with STS modes of understanding (Walford 2013). Ironically, however, problems also arise if the concepts and interpretations of our interlocutors seem "too similar" to our own (Riles 2000).

In one sense, the lateral designates the broad observation that domains of knowledge and practice influence each other in unpredictable ways. At this level, the lateral can be seen as a "meta-concept." In one sense, therefore, our note can be read as a generic characterization of how concepts are generated.

In another sense, however, lateral concepts are entirely immanent to particular inquiries. This is because their development begins with the recognition of specific kinds of movement between forms of knowledge within a particular field of concern. If movements and modifications of forms of knowledge happen continuously, the lateral question becomes one of activation: how might researchers draw energy from something that is happening in front of our eyes anyway. At this level, lateral conceptualization is thus thoroughly empirical. We might even say that it is radically empirical, in that concepts and theories are seen to be located within the empirical field itself.

Provisionally, we might thus define lateral conceptualization as efforts at concept development and improvement that work on the basis of a mutual modification of STS (or otherwise "academic") concepts and the varied concepts and ideas encountered in the field. Further on, we offer some examples of what this might mean.

We begin, however, with a brief introduction to lateral anthropology. Because this "approach" emerged as a sympathetic yet critical counter-point to actor-network theory, we subsequently turn to Latour's (1988) argument for "infra-reflexivity," in order to figure out just what lateral analysis reacted against. We end by illustrating some ways in which lateral conceptualization enables a reorientation of STS modes of inquiry.

"For example, it is by no means given that they must inevitably take the form of conventional class, race and gender differences as routinely assumed by those who critique Latour in the name of "politics." 


\section{Lateral Anthropology}

In The Gender of the Gift, Marilyn Strathern (1988) insisted that anthropological analysis would have to take its starting point in indigenous conceptualization. She was clear, however, that it would have to do so without simply adopting "found" concepts. In later works (1992a, 1992b), she used notions of Melanesian personhood as a lever for the analysis of English kinship. The lateral is exhibited in both of these moves. First, Strathern loosened the relation between "our" concepts and "theirs." Second, she positioned them as contexts for each other, deploying Melanesian ideas as a resource for rethinking British ones and vice versa.

Strathern did not use the notion of the lateral. However, the anthropologist Bill Maurer (2005) referred explicitly to his study of Islamic banking and alternative currencies as a deployment of "lateral reason" (see also e.g. Helmreich 2011). Pointing out that ethnographic sites are already overflowing with peoples' concepts and comparisons, Maurer asked how found concepts might be allowed to make a difference for anthropological analysis. Dispensing with the aspiration to offer anthropological meta-explanations that would "trump" those encountered in the field, he located social science knowledges alongside those they investigate. Thus, his analysis "revealed the tropes of Islamic Banking, alternative currencies, and my inquiry as open and unsteady" (2005: 11). The traffic of concepts was seen to run in multiple directions.

Strathern's former student, Annelise Riles made similar arguments. Her emphasis was especially on situations where "our" knowledges and those of informants are in some sense too close. New kinds of anthropological response are called for, she suggested, in contexts where the distance between "our" understandings and "theirs" threatens to collapse. If, for example, NGO workers already conceive their activities in terms of networks, what is analytically gained by offering a network analysis of them? In this situation, an unqualified acceptance of informants' self-understandings would merely reinforce what we think we already know.s What is needed in this situation are devices for estrangement rather than for familiarization. Thus, for example, Riles turned practices of Fijian mat making into a conceptual and comparative device for the interpretation of policy documents (2000: 70ff).

As this example indicates, lateral reason does not impose any fixed obligation on the researcher. After all, nothing demanded that Riles used mats to understand policy. At issue,

"It can be argued, however, that Riles maintains a rather generic distinction between "us" and "them" throughout The Network Inside Out. 
instead, is an experimental opening up of resources for thinking. The wager is that such materials hold the capacity for troubling or reorienting conventional theory in productive ways if given the chance.

As noted, lateral reason has been developed in explicit response to actor-network theory. In contrast with the realism and empiricism of the latter (sometimes perceived, sometimes avowed), Strathern, Riles and Maurer insist on inventiveness as a key ingredient of ethnographic description (Jensen 2012). Maurer, for example, comments critically on an undercurrent of empiricist realism that he perceives within ANT:

Latour's realism is problematic for the same reason that it is so useful: refusing the separation of epistemology from ontology opens up the enumerable black boxes that warrant "reality," but it does so in terms of that refusal's own agnosticism (Maurer 2005:

14).

The empiricism and realism to which Maurer alludes is captured by Latour's famous slogan "follow the actors," later morphed into the metaphor of the STS scholar as an "ant" that meticulously traces naturally occurring relations between heterogeneous actors. Yet, it is only possible to claim the ability to depict reality as such, if the researcher is capable of neutrally registering it. Maurer is asking us to consider whether the "ant" is indeed able to miraculously evade the problems of representation that have haunted anthropology since the 1980s.

At this point, then, there is a direct point of contact between lateral anthropology and STS, at least in its ANT guise. To understand the workings of lateral concepts, let us therefore examine this contact zone in a bit more detail.

\section{Infra-reflexive Ambiguities}

In the 1980s, anthropology was grappling with the so-called crisis of representation (Clifford and Marcus 1986). At issue were the (im-)possibility and political implications of representing "the other." One outcome was new forms of reflexive ethnographic writing. Something similar was afoot in science and technology studies. In Knowledge and Reflexivity, Steve Woolgar (1988) argued that STS too was haunted by a lingering tendency to descriptivism and objectivism. Maurer's critical comments on Latour's "agnosticism" offer an updated version of this kind of critique. 
In some ways analogous to Writing Culture, Woolgar's solution was to write multilayered and self-reflexive texts. These experimental efforts would circumvent the possibility that readers would take the text for a "correct" representation of its object.

Bruno Latour also contributed to Knowledge and Reflexivity. However, rather than following the path suggested by Woolgar, his chapter offered a critique of the volume's reflexive ambition. Referring to this ambition as "meta-reflexive," Latour argued that it leads to an endless spiral of self-referential commentary in which the substance of inquiry recedes from view.

Even so, self-reference does not enable the writer to solve any problems of representation. Because the writer operates in a representational space as soon as $\mathrm{s} /$ he puts pen to paper, Latour argues, there is no "problem" to be overcome in the first place. What metareflexivity does is simply to tip the textual balance, so that more of the writer's energy goes into making visible the workings of the text itself rather than its supposed subject. Latour's infrareflexive alternative is concerned, instead, with figuring out how to convey in writing bits of reality "imported" from outside the text:

Instead of piling on layer upon layer of self-consciousness, why not have just one layer, the story, and obtain the necessary amount of reflexivity from somewhere else? (Latour 1988: 170)

There is a certain ambiguity here. The "somewhere else" invoked by Latour might refer to the intellectual resources and stylistic devices which all writers, including "journalists, poets, and novelists" (170) deploy to make their texts "good" or "convincing." But in the case of journalists and ethnographers, there is a complex relation between those resources and the materials encountered in the field.

As we have noted, the ambiguous relation between writing and field is the very signature of lateral concepts as we defined them above. Yet Latour wavers about the implications of the ambiguity. Thus, he has also argued that the most suitable role for ANT researchers is to operate as "ants" that simply "trace" the world's contours. When Maurer complains about Latour's agnosticism and Riles insists on the importance of "invention," they are reacting to this empiricist claim.

The situation is therefore complicated. As anyone who has read Latour's own works knows, far from merely "tracing" the activities and relations of people and things, he is engaged in a constant conceptual labor. In this light, Latour's insistence that other scholars should simply 
"trace" reality appears disingenuous. At the same time, as we have seen, "infra-reflexivity" is clearly not an argument for any simple replacement of conceptual and stylistic experimentation with found reality. Quite differently, it designates a kind of open-ended invention in which analysts' and informants' concepts and practices both inform the textual re-description of realities. We can thus read the "infra-reflexivity" as an STS precursor of lateral analysis.

If it is nevertheless only a precursor, the reason is precisely Latour's tendency to withdraw from the lateral entailments of the argument and hide behind the discourse of empirical tracing. For if the "somewhere else" in the end comes to designate a field to be simply traced by ants, then what role might the stylistic and conceptual experimentation he also encourages possibly play? Rather than evading this problem, lateral conceptualization aims to activate it.

\section{Activations}

When STS ethnographers explore their fields, they are not the only "knowers," for their "informants" are involved in their own knowledge practices. Since these concepts are shaped and transform through complex interactions with those of other informants, sometimes including social theorists, the "field" elicits lateral characteristics. What to do about this situation? Various possibilities suggest themselves.

For one, it is possible to simply ignore the lateral predicament. This is exemplified by the routine application of theories to explain "the empirical." In such cases, the conventional dichotomy between theory and practice, desk and field, is in no way challenged. In contrast, lateral conceptualization is premised on the potentials of working with these lateral movements of divergent forms of knowledge; to activate them and let them interfere with standard analytical forms (Gad 2012, Gad and Jensen 2010, 2016).

To go down the lateral route is not necessarily an easy decision, though, for in doing so one loses epistemic superiority. What is gained, however, is an experimental opening to other forms of encounters and concepts. At stake is a fundamental deregulation of how concepts and ideas are allowed to travel and interact. The name of the game cannot simply be empirical ("just follow the actors"), and the lateral certainly does not mean that theory becomes a "bad word" ("just leave the ivory tower and listen to what your informants are saying").

Symmetrically, however, lateral analysis also eschews the application of ready-made concepts and theories that do not run the risk of modification through worldly encounters. Since 
both research practices and informants' practices are involved in making knowledge, we might say that the lateral makes everything empirical. Since STS concepts and found concepts are put in conjunction, we might equally say that the lateral makes everything conceptual. Either way, the lateral sets in motion a dynamic reconfiguration of what it means to do analysis.

But why one would voluntarily give up epistemological privilege? What is gained by doing so? The ethical response is that it is a way of taking people's understandings seriously, rather than submerging them under our own pre-established theoretical categories. The analytical or conceptual response is that doing so makes it possible to shift or redefine questions premised on the sharp dichotomy between the conceptual and the empirical. We end this note by offering two brief examples of how that works.

The first comes from Atsuro Morita's (2014) excellent analysis of Thai mechanics working with Japanese second-hand harvesting machines in Northern Thailand. These machines often break down as farmers begin using them in their fields. It is the job of mechanics to fix them. As good practitioners, they are thus involved in the practical modification of machines. But as Morita shows, in order to succeed in this task, they use the broken machines as comparative devices for understanding the difference between Thai and Japanese fields. These comparisons generate a perspective on what Japanese fields look like, which is then used to imagine the modifications required for the machines to function under Thai conditions.

This ethnography could easily go down the path of examining the "local, situated practices" of Thai farmers and mechanics. By lateralizing the analysis, however, Morita moves in quite a different direction. Rather than focusing exclusively on practice, he uses the comparative labor of the mechanics, and their resulting understanding of the difference between Japanese and Thai fields, as his conceptual starting point. This allows him to challenge conventional anthropological notions of Thai culture as well as to offer a new interpretation of the problems faced by "technology transfer" initiatives, which are often premised on the idea that the recipients of the transfer are local practitioners lacking trans-local knowledge. Furthermore, Morita's comparative adaptation of the machine-generated perspectives of Thai mechanics enables him to re-describe ethnography itself as a machine for the articulation of surprising comparisons and connections.

Our own previous research offers another example of the analytical potential of lateral conceptualization. While studying the relation between national and EU fisheries regulation and practices of fisheries inspection at sea, Christopher grappled with the question of how to characterize the culture of sailors and "life at sea." Of course, many aspects of such life could be 
observed "directly" while doing fieldwork onboard an inspection vessel. Surprisingly, however, when the crew was asked about their "culture," they directed him to a 1960s movie, Martha, that had received a cult following among sailors in Scandinavia (Gad 2012). As far as they were concerned, this movie was far more expressive of their "culture" than their own practices and verbalizations.

Of course, just as nothing forced Riles to compare Fijian mats to policy documents, nothing required Christopher to engage with this unlikely explanatory source. Yet, positioning the movie laterally in relation to the field, as offering a "theory of culture," created certain analytic openings.

Depicting a crew of sailors enjoying life in the Mediterranean on a rusty old ship almost forgotten by its owners, Martha dramatized an ideal of the "free" life of seamen. Moreover, it showed a struggle between the traditional virtues of seamanship and novel forms of technological modernity that threatened those virtues. It also exhibited sailors sufficiently independent and strong-minded to "man up" and square the challenges of modern business with its emphasis on technological efficiency, control and management.

Precisely by dramatizing an image of life at sea as it never really was, the movie made it possible to grasp the sailors' felt importance of independence and freedom, which was considerably less visible in their everyday inspection practice. Moving laterally from inspection practices to the self-image of sailors as evinced by Martha, thus made it possible for Christopher to articulate the paradoxical situation in which inspectors found themselves: at once sailors congenially unwilling to delimit the independence of other sailors (as one said "it is against my nature to control others") and government bureaucrats working in a heavily regulated and technologized environment to do precisely that.

Moreover, the significance attached by the sailors to the cult movie necessitated a lateral extension of the idea of infra-reflexivity. For even as references to the movie occurred in particular encounters onboard the ship, the source of reflexivity was not found in "local practice." Analogous to the Thai mechanics, whose practices drew inspiration from the "elsewhere" of Japanese fields they had never visited, local practice on board the ship was reflexively sustained with reference to the "somewhere else" of a past that never existed. No more confined to "local practice" than STS scholars, these cases indicate that our informants deploy "inter-reflexive" resources found outside the spatial and temporal confines of their own practices. Reflexivity is as much inter- as infra, as it emerges in encounters across contexts or networks (see also Ratner 2013). 
In this brief note we have shown that lateral concepts offer fresh analytical opportunities once it is granted that both researchers and those they study are fundamentally decentered. The risks and possibilities of the lateral, therefore, are about nurturing an attention to what it takes to establish relative forms of compatibility between divergent forms of knowing and acting in such a decentered world.

\section{References}

Clifford, J. and Marcus, G. E. (Eds). 1984. Writing Culture: The Poetics and Politics of Ethnography. Berkeley, Los Angeles and London: University of California Press.

Gad, C. (2012) "What we Talk about when we Talk about Sailor Culture: Understanding Danish Fisheries Inspection through a Cult Movie," Culture Unbound 4: 367-92.

Gad, C. and C. B. Jensen. (2010) "On the Consequences of Post-ANT," Science, Technology and Human Values 35(1): 55-80.

Gad, C. and C. B. Jensen. (2016) "Lateral Comparison." In Practicing Comparison: Logics, Relations, Collaborations, edited by J. Deville, M. Guggenheim and Z. Hrdličková. Mattering Press.

Gad, C., and C. B. Jensen. and B. R. Winthereik. 2015 "Practical Ontology: Worlds in STS and Anthropology," NatureCulture 3: 67-86.

Helmreich, S. 2011. "Nature/Culture/Seawater," American Anthropologist 113(1): 132-44.

Jensen, C. B. 2012. "The Task of Anthropology is to Invent Relations," Critique of Anthropology 32(1): 47-53.

Jensen, C. B. 2014. "Continuous Variations: The Conceptual and the Empirical in STS," Science, Technology and Human Values 39(2): 192-213.

Latour, B. 1988. "The Politics of Explanation." In Knowledge and Reflexivity, edited by S. Woolgar, 155-77. London: Sage.

Maurer, B. 2005. Mutual Life, Limited: Islamic Banking, Alternative Currencies, Lateral Reason, Princeton, NJ: Princeton University Press.

Morita, A. 2014. "The Ethnographic Machine: Experimenting with Context and Comparison in Strathernian Ethnography," Science, Technology and Human Values 39(2): 214-235.

Ratner, H. 2012. Promises of Reflexivity: Managing and Researching Inclusive Schools. PhD thesis, Copenhagen Business School.

Riles, A. 2000. The Network Inside Out. Ann Arbor, MI: University of Michigan Press.

Strathern, M. 1988. The Gender of the Gift: Problems with Women and Problems with Society in Melanesia. Berkeley, Los Angeles and London: University of California Press.

Strathern, M. 1992a. After Nature: English Kinship in the Late Twentieth Century. Cambridge: Cambridge University Press.

Strathern, M. 1992b. Reproducing the Future. New York: Routledge.

Walford, A. C. 2013. Transforming Data: An Ethnography of Scientific Data from the Brazilian Amazon. $\mathrm{PhD}$ thesis. IT University of Copenhagen.

Woolgar, S. (Ed.) 1988. Knowledge and Reflexivity. London: Sage. 